\title{
11 AS CRIANÇAS E JOVENS REFERENCIADAS À CPCJ: O ESPELHO DAS PROBLEMÁTICAS
}

\author{
$\mid$ Aida Correia Simões ${ }^{1}$; Maria dos Anjos Coelho Dixe²; Maria Saudade Lopes ${ }^{3}$
}

\section{RESUMO}

CONTEXTO: As crianças e jovens referenciadas à CPCJ: O espelho das problemáticas.

OBJETIVOS: Os objetivos deste estudo foram: identificar as problemáticas e quem mais sinaliza as situações de crianças e jovens em rico/perigo, verificar se as problemáticas envolvidas na sinalização de crianças e jovens em risco mudaram da primeira para a segunda sinalização e caracterizar as famílias das crianças e jovens em risco/perigo.

METODOLOGIA: Estudo descritivo de abordagem quantitativa resultante da consulta de 160 processos.

RESULTADOS: Dos 160 casos - 41,9\% constituída por famílias nucleares (pais e filhos) e 20\% mononucleares (mãe e filhos) - a negligência foi a principal causa de sinalização, não só na primeira sinalização $(38,5 \%)$ como na reabertura (26,3\%). Na reabertura do processo verificamos que um grande número mantém a mesma problemática, ou seja, $29 \%$ negligência, $25 \%$ comportamentos desviantes, $20 \%$ absentismo escolar; abandono e absentismo escolar são igualmente causas com elevada prevalência não só na primeira sinalização como na reabertura do processo. A escola é a entidade que mais sinaliza: $34,4 \%$ na $1^{\text {a }}$ sinalização e $46,5 \%$ na $2^{\text {a }}$ sinalização; em $71,3 \%$ dos casos a criança não é seguida por um profissional de saúde mental e psiquiátrica e 10\% (16) é seguida pela pedopsiquiatria, $0,6 \%$ (1) por psiquiatra e igual número por um serviço de internamento e finalmente $17,5 \%$ (28) são acompanhadas por um psicólogo. Quanto aos pais verificou-se que 85,8\% (139) não tem qualquer acompanhamento; 9,3\% (15) dos casos somente a mãe tem acompanhamento psiquiátrico; $1,2 \%$ (2) dos casos somente o pai e em $0,6 \%$ (1) dos casos ambos os pais são acompanhados por um psiquiatra. 3,1\% (5) não referiram quem os acompanha. CONCLUSÃO: No artigo 27 da Convenção dos Direitos da Criança podemos ler que esta deve beneficiar de um nível de vida adequado ao seu desenvolvimento, ao confrontarmos esta afirmação com os nossos resultados podemos verificar que dos processos reabertos pela CPCJ a problemática de perigo que mais se evidência é a negligência, uma grande percentagem das crianças/jovens coabita nas suas famílias nucleares, para além disso da $1^{\mathrm{a}}$ para a $2^{\mathrm{a}}$ sinalização não se verifica nenhum tipo de acompanhamento na área da saúde mental, a nenhum dos elementos do agregado familiar, o que poderá contribuir para que a situação se perpetue no tempo e não havendo mudança de comportamento torna-se inevitável referenciar novamente a criança/jovem.

\section{PALAVRAS-CHAVE: Crianças e jovens em perigo; Comissões de proteção; Problemáticas}

\section{RESUMEN}

\section{"Los niños y jóvenes mencionados a la Comisión de Protección de niños y jóvenes (CPCJ): El espejo de las problemáticas"}

CONTEXTO: Los niños y jóvenes mencionados a la Comisión de Protección de niños y jóvenes (CPCJ): El espejo de las problemáticas

OBJETIVOS: Los objetivos de este estudio fueron identificar el problema y quién más señala la situación de los niños y jóvenes en ricos/peligro, verificar se las cuestiones implicadas en la señalización de los niños y jóvenes en situación de risco, ha cambiado desde la primera a la segunda señalización y caracterizar las familias de los niños y la juventud en situación de riesgo / peligro.

METODOLOGÍA: Estudio descriptivo de enfoque cuantitativo resultante de la consulta de 160 casos.

RESULTADOS: De los 160 casos - 41,9\% formado por familias nucleares (padres y niños) e $20 \%$ mononucleares (madre e hijos) - negligencia fue la causa principal de la señalización no sólo en la primera referencia $(38,5 \%)$ como en la reapertura $(26,3 \%)$; se constató que en la reapertura del caso gran número sigue teniendo el mismo problema, es decir, el $29 \%$ el abandono, el $25 \%$ un comportamiento desviado, un $20 \%$ el absentismo escolar. La deserción escolar y el absentismo son también causa alta prevalencia no sólo en la primera señalización como en la reapertura del caso; Escuela es la entidad que hace la mayoría de las señalizaciones: $34,4 \%$ en el primero de señalización y $46,5 \%$ en el segundo de señalización. En 71,3\% de los casos el niño no es seguido por un profesional de salud mental y psiquiátrica, $10 \%$ (16) es seguido por la psiquiatría infantil, 0,6\% (1) por un psiquiatra y un número igual por un servicio de internamiento y, finalmente, $17,5 \%$ (28) son seguidos por un psicólogo. Para los padres se encontró que 85,8\% (139) no tiene vigilancia; $9,3 \%$ (15) de los casos sólo la madre tiene la atención psiquiátrica; $1,2 \%$ (2) de los casos sólo el padre y el 0,6\% (1) de los casos ambos padres son acompañados por un psiquiatra. $3,1 \%$ (5) no informaron quien los acompaña.

CONCLUSIÓN: En el artículo 27 de la Convención de los Derechos del Niño leemos que el niño debe tener un nivel de vida adecuado para su desarrollo, confrontando esta declaración con nuestros resultados podemos ver que en los procesos abiertos de nuevo por la CPCJ lo problema de riesgo más evidente es negligencia, un gran porcentaje de niños / jóvenes que cohabitan en sus núcleos familiares, además de la primera a la segunda señalización no ocurra ningún tipo de supervisión en el área de la salud mental de cualquiera de los miembros del hogar, lo que puede contribuir a la situación se perpetúa en el tiempo y si no hay ningún cambio de comportamiento se vuelve inevitable referenciar de nuevo al niño / joven.

\section{DESCRIPTORES: Niños y jóvenes en peligro; Comisión de protección; Problemáticas}

\section{ABSTRACT \\ "Children and young people referenced to the Child and Youth Protection Commission (CPCJ): The problematics mirror"}

BACKGROUND: Children and young people referenced to the Child and Youth Protection Commission (CPCJ): The problematics mirror.

AIM: The objectives of this study were to identify the problem and who else signals the situations of children and young people in rich / danger, ensure that the issues involved in the signaling of children and youth at risk, has changed from the first to the second signal and characterize the families of children and youth at risk / danger.

METHODS: Descriptive study of quantitative approach resulting from 160 cases consultation.

RESULTS: Of the 160 cases - 41.9\% made up of nuclear families (parents and children) and $20 \%$ mononuclear (mother and children) - negligence was the main cause of signaling, not only the first signaling $(38.5 \%)$ but in reopening cases $(26.3 \%)$. On the reopening of the cases we found that a large number remains with the same problems, which are, $29 \%$ neglect, $25 \%$ deviant behavior, $20 \%$ school absenteeism. School dropout and absenteeism are also high prevalence causes, not only in the first signaling as in the case reopening. The school is the entity that most signals: $34.4 \%$ at the 1 st signaling and $46.5 \%$ at the 2 nd; in $71.3 \%$ of cases the child is not followed by a mental and psychiatric health specialist and $10 \%(16)$ is followed by child psychiatry, $0.6 \%$ (1) by a psychiatrist and an equal number for an inpatient service and finally $17.5 \%$ (28) are accompanied by a psychologist. The parents were found to be $85.8 \%$ (139) has no monitoring; $9.3 \%$ (15) of the cases only the mother has psychiatric care; $1.2 \%$ (2) of the cases only the father and $0.6 \%$ (1) cases both parents are accompanied by a psychiatrist. $3.1 \%$ (5) did not report who accompanies them.

CONCLUSION: On the Article 27 of the Child Rights Convention we can read that the child should benefit from an adequate standard of living for its development, by confronting this statement with our results we can see that in the processes reopened by CPCJ the most evidence hazard problem is neglect, a large percentage of children / young people cohabiting in their nuclear families, furthermore from 1 st to the 2 nd signaling did not happen any kind of mental health follow-up for none of the household members, which may contribute to the persistence of the situation and if there is no behavior changes becomes inevitable reference the child/ young again.

\section{KEYWORDS: Children and young in danger; Protection com- mission; Problematics}

Submetido em 25-11-2015

Aceite em 10-03-2016

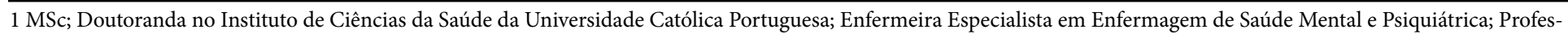
sora Adjunta na Escola Superior de Enfermagem Egas Moniz, Via Alternativa ao Monte da Caparica, 2829-511 Caparica, Portugal, aidasimoes@gmail.com

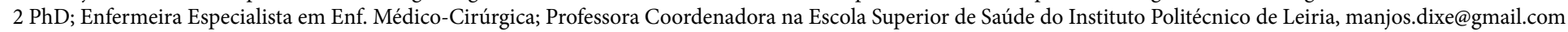

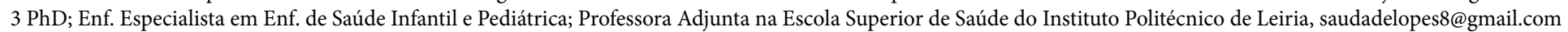

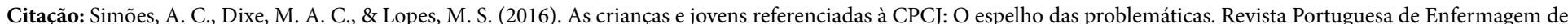
Saúde Mental (Spe. 3), 63-66. 


\section{INTRODUÇÃO}

As Comissões de Proteção de Crianças e Jovens (CPCJ) constituem uma estrutura de segunda linha no sistema de promoção e proteção das crianças em situação de perigo (Leandro, 1999). Para o problema de desenvolvimento que Portugal e o Mundo vivem só pode haver resposta "no desenvolvimento da pessoa", disse, sublinhando que não é possível o progresso sem que na sua base esteja o sujeito. O presidente da CNCJR (Comissão Nacional de Proteção de Crianças e Jovens em Risco), Armando Leandro referiu numa notícia do DN a importância das comunidades locais, como territórios "com uma história e um futuro que querem construir", na tarefa da prevenção, num país em que essa cultura é descurada nas mais diversas áreas, sobretudo numa área exigente como é a dos direitos das crianças. "Vivemos tempos difíceis mas não podemos conformar-nos, muito menos porque as crianças sofrem com esta realidade", disse, sublinhando a importância dos afetos, que é hoje um direito como são a educação para o respeito e a paz, tão importantes em problemas de comportamento e de disciplina (Robalo, 2014).

As reaberturas processuais implicam a existência de crianças/jovens que são referenciados várias vezes no decorrer do seu percurso de desenvolvimento. As sinalizações nem sempre obedessem à mesma problemática, diz-nos a literatura que crianças negligênciadas são jovens com maior tendência para apresentar comportamentos desviantes, com consumos de alcool e drogas, abandonarem a escola e não se enquadrarem na sociedade em que estão inseridas.

\section{METODOLOGIA}

Estudo descritivo baseado nos registos de 160 processos de crianças /jovens sinalizadas pelo menos duas vezes (reabertura do processo). Os principais objetivos deste estudo foram Identificar as problemáticas das reaberturas dos processos de crianças/jovens e quem mais sinaliza as situações de crianças e jovens em rico/perigo, verificar se as problemáticas envolvidas na sinalização de crianças e jovens em risco, mudou da primeira para a segunda sinalização e caracterizar as famílias das crianças e jovens em risco/perigo. Para a consulta dos processos, realizada em 2014, foi solicitada autorização e respeitados os princípios éticos inscritos na declaração de Helsínquia.

Foi utilizado o programa SPSS para tratamento de dados e utilizada a estatística descritiva para a sua análise.

\section{RESULTADOS}

Dos 160 casos, 59,3\% das crianças/jovens sinalizados eram do sexo masculino e com uma média de idades

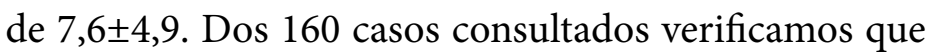
a constituição familiar era em $41,9 \%$ constituída por famílias nuclear (pais e filhos), 20\% mononucleares (mãe e filhos). Da primeira para a segunda sinalização a estrutura familiar alterou-se, ou seja, 28,6\% eram famílias nucleares e 15,5\% monoparentais. Apesar de ser pouco expressivo, em todas as sinalizações há crianças que vivem com os tios $(3,1 \%)$ e com os avós $(16,1 \%)$. A negligência foi a principal causa da sinalização não só na primeira sinalização $(38,5 \%)$ como na reabertura $(26,3 \%)$ e não depende da tipologia familiar (constituição). De salientar que o abandono e absentismo escolar são igualmente causas com elevada prevalência não só na primeira sinalização como na reabertura do processo (Tabela 1).

Tabela 1 - Problemática da primeira, segunda e terceira sinalização

\begin{tabular}{|l|l|l|l|l|l|l|l|}
\hline \multirow{2}{*}{ Tipologia das Situações de Perigo } & \multicolumn{2}{|l|}{ Sinalizac̃o 1} & \multicolumn{2}{|l|}{ Sinalizac̃o 2 } & \multicolumn{2}{|l|}{ Sinalizã̃o 3 } \\
\hline & $\mathrm{N}^{\circ}$ & $\%$ & $\mathrm{~N}^{\circ}$ & $\%$ & $\mathrm{~N}^{\circ}$ & $\%$ \\
\hline Negligência & 62 & 38,5 & 42 & 26,3 & 6 & 28,6 \\
\hline Roubo & 1 &, 6 & & & & \\
\hline Violência domestica & 6 & 3,7 & 3 & 1,9 & 1 & 4,8 \\
\hline Rapto do pai & 2 & 1,2 & & & & \\
\hline maus tratos psicológicos & 6 & 3,7 & 11 & 6,9 & & \\
\hline Maus tratos físicos & 19 & 11,8 & 8 & 5,0 & 1 & 4,8 \\
\hline Problemas de saúde & 2 & 1,2 & & & & \\
\hline Comportamentos desviantes & 8 & 5,0 & 20 & 12,5 & 2 & 9,5 \\
\hline Gravidez na adolescência & 3 & 1,9 & 1 &, 6 & & \\
\hline Maus tratos físicos e psicológicos & 2 & 1,2 & & & & \\
\hline Absentismo escolar & 15 & 9,3 & 25 & 15,6 & 2 & 9,5 \\
\hline Abandono escolar & 17 & 10,6 & 18 & 11,3 & 7 & 33,3 \\
\hline Abandono dos pais & 1 &, 6 & & & & \\
\hline Abandono da mãe & 3 & 1,9 & 2 & 1,3 & & \\
\hline Abandono & 5 & 3,1 & & & & \\
\hline Buling & 1 &, 6 & & & & \\
\hline Dificuldades económicas & 3 & 1,9 & 7 & 4,4 & & \\
\hline Abuso Sexual & 1 &, 6 & 3 & 1,9 & & \\
\hline Agressão efetuado pelo menor & 1 &, 6 & 2 & 1,3 & & \\
\hline Família disfuncional & 2 & 1,2 & & & & \\
\hline $\begin{array}{l}\text { Doença mental/psiquiátrica- } \\
\text { bipolar }\end{array}$ & 1 &, 6 & & & & \\
\hline Morte da mãe & 3 & 1,9 & 3 & 1,9 & & \\
\hline $\begin{array}{l}\text { Exposição a comportamentos } \\
\text { desviantes }\end{array}$ & 5 & 3,1 & & & 2 & 9,5 \\
\hline Maus tratos físicos padrasto & & & 4 & 2,5 & & \\
\hline $\begin{array}{l}\text { Maus tratos físicos por parte } \\
\text { do pai }\end{array}$ & & & 1 &, 6 & & \\
\hline Abuso sexual padrasto & & & 1 &, 6 & & \\
\hline Regulação parental & & & 1 &, 6 & & \\
\hline Paradeiro desconhecido & & & & & \\
\hline Responsabilidades. Parentais & & & & \\
\hline
\end{tabular}


Para a reabertura do processo verificamos que um grande número mantém a mesma problemática, ou seja, $29 \%$ negligência, $25 \%$ comportamentos desviantes, $20 \%$ absentismo escolar.

Foram sinalizadas duas vezes $88,0 \%$ (140) das crianças/ jovens, três vezes $11,9 \%$ (19) crianças.

É a escola a entidade que mais sinaliza: $34,4 \%$ na $1^{\text {a }}$ sinalização e $46,5 \%$ na $2^{\text {a }}$ sinalização. De salientar no entanto, que os vizinhos, avós e as IPSS (centro comunitário) são igualmente as entidades que mais sinalizam a negligência.

Em $71,3 \%$ dos casos a criança não é seguida por um profissional de saúde mental e psiquiátrica e 10\% (16) é seguida pela pedopsiquiatria, $0,6 \%$ (1) por psiquiatra e igual número por um serviço de internamento e finalmente 17,5\% (28) são acompanhadas por um psicólogo. Relativamente ao acompanhamento dos pais por um profissional de saúde mental e psiquiátrico, verificou-se que $85,8 \%$ não tem qualquer acompanhamento e 9,3\% (15); pai $1,2 \%$ (2); pai e mãe $0,6 \%$ (1); são acompanhados por um psiquiatra, sendo que 3,1\% (5) não referiram quem os acompanha.

Os gestores de caso são essencialmente maioritariamente os S. social (32,3\%), CMP $(29,8 \%)$ e os serviços de educação $(22,4 \%)$.

\section{DISCUSSÃO}

Segundo Camilo e Garrido (2013) a negligência parental tem surgido associada à falta de competência dos pais para educar, supervisionar e responder às necessidades dos filhos menores, colocando em risco o seu bem-estar, as mesma autoras referem que são ainda escassos os programas de intervenção para cuidadores devidamente estruturados, metódicos e estratégicos bem definidos, sendo raros os que utilizam desenhos de avaliação que permitam aferir adequadamente a sua eficácia.

A vulnerabilidade psicossocial a que estas famílias estão sujeitas associa-se muitas vezes a riscos para o bemestar dos seus membros, nomeadamente das crianças e jovens. A negligência parental constitui uma dessas vulnerabilidades, fundamentando assim a necessidade de intervenções que a par do suporte socioeconómico do Estado, potenciem as competências parentais das mesmas (Camilo \& Garrido, 2013). Em alguns estudos é possível verificar que os programas de competências parentais contribuem para a prevenção da negligência, diminuindo assim, os comportamentos negligentes e maltratantes, ainda de acordo com o mesmo estudo verifica-se uma melhoria das condições habitacionais, ao nível da higiene e da alimentação (Canedo, 2014).
Se consultarmos o artigo 27 da Convenção dos Direitos da Criança podemos ler que esta deve beneficiar de um nível de vida adequado ao seu desenvolvimento, ao confrontarmos esta afirmação com os nossos resultados podemos verificar que dos processos reabertos pela CPCJ a problemática de perigo que mais se evidência é a negligência, uma grande percentagem das crianças/ jovens coabita nas suas famílias nucleares, para além disso da $1^{\text {a }}$ para a $2^{\mathrm{a}}$ sinalização não se verifica nenhum tipo de acompanhamento na área da saúde mental, a nenhum dos elementos do agregado familiar, o que poderá contribuir para que a situação se perpetue no tempo e não havendo mudança de comportamento torna-se inevitável referenciar novamente a criança/jovem. Segundo vários autores o desenvolvimento de competências sociais educativas dos pais pode ajudar na prevenção de problemas de comportamento das crianças, dado que, problemas de comportamento estão direta e/ou indiretamente relacionados com o desígnio comportamental dos pais. (Bolsoni-Silva \& Del Prette, 2002; Weber, Prado, Viezzer \& Bandenburg, 2004). A questão da educação é outra das grandes preocupações, como podemos verificar nos resultados que obtivemos. Esta é uma tipologia de perigo que tem vindo a aumentar desde que a escolaridade se tornou uma obrigatoriedade até aos 18 anos de idade, dados da Unicef em 2007, já constatavam que $30 \%$ dos nossos jovens deixavam os estudos e $23 \%$ destes não tem emprego, fazendo-nos lembrar que "correm indubitavelmente maior risco de exclusão e marginalização" (Unicef, 2007).

\section{CONCLUSÃO}

Mais de metade das crianças sinalizadas são do sexo masculino, pertencentes maioritariamente a famílias nucleares e tal como noutros estudos o maior percentual de sinalização é a negligência, mantendo se a mesma cauda para a reabertura dos processos. Dado que o absentismo escolar apresenta também um elevado percentual de sinalização é a escola que mais sinaliza.

Continuaremos a questionar que futuro se está a construir para as nossas crianças/jovens, que saúde mental temos para lhes oferecer, como vamos conseguir inverter esta pirâmide cuja prevenção se torna cada vez mais estreita e inexistente. 
